\title{
Well-differentiated liposarcoma of the hypopharynx: Two case reports and literature review
}

\author{
Maria Köping'*, Rudolf Hagen ${ }^{1}$, Schneider Michael ${ }^{2}$, Christopher Beck ${ }^{2}$,Jan-Peter Grunz ${ }^{3}$ and Agmal Scherzad $^{1}$ \\ ${ }^{1}$ Department of Oto-Rhino-Laryngology, Plastic, Aesthetic and Reconstructive Head and Neck Surgery, University Hospital Würzburg, Josef-Schneider-Strasse 11, \\ D-97080 Würzburg, Germany \\ ${ }^{2}$ Department of Pathology, University of Würzburg, Josef-Schneider-Strasse 2, D-97080 Würzburg, Germany \\ ${ }^{3}$ Department of Diagnostic and Interventional Radiology, University Hospital Würzburg, Oberdürrbacher Strasse 6, 97080 Würzburg, Germany
}

\begin{abstract}
The well-differentiated liposarcoma of the hypopharynx is an extremely rare tumor entity. Symptoms can include dysphagia, globus sensation up to asphyxia due to laryngeal obstruction. This is a report on two cases of enormous liposarcomas originating from the hypopharynx and the diagnostic and therapeutic approach. There were neither nodal nor distant metastases, complete resection is essential to avoid local recurrence. The liposarcoma should be kept in mind in patients with unclear proliferation in the hypopharyngeal area.
\end{abstract}

\section{Introduction}

The liposarcoma is a common soft tissue sarcoma arising from adipose tissue [1,2]. Mostly, it is localized in the extremities and retroperitoneum. In less than $6 \%$, it is in the head and neck region [3]. With less than 30 cases in literature occurrence in the hypopharynx is extremely rare [4-6]. Due to its localization and progressive growth patients complain about dysphagia, globus sensation or hoarseness. Nevertheless also, a critical to life-threatening airway obstruction could develop.

Pathohistologically, liposarcomas are divided into five subtypes [7]: well-differentiated, myxoid, pleomorphic, round cell and dedifferentiated. The well-differentiated liposarcoma is the most common histopathological type. In some subtypes of liposarcoma the sequences of chromosomal region 12q14-15 which contains the murine double-minute 2 gene (MDM2) is amplified. Thus, the most useful tool in the diagnosis of liposarcoma is the fluorescent in-situ hybridization (FISH) mediated detection of MDM2 gene amplification [8].

This is a report on two cases of enormous liposarcoma originating from the hypopharynx and the diagnostical and surgical procedure, including a literature review on previous publications.

\section{Case 1}

A 38-year old woman presented in Mai 2018 with progressive dysphagia and globus sensation with a weight loss of $10 \mathrm{~kg}$. Dyspnea was denied. In the transnasal endoscopy a slight salivary retention in the right piriform sinus was found. An FDG-PET/CT scan showed a great inhomogeneous, partly hypermetabolic intraluminal tumor of the proximal two thirds of the esophagus, as well as several hypermetabolic, most likely reactive lymph nodes in the left jaw angle (Figure 1A). MRI revealed a solid and partly fatty tumor without invasion of the esophageal wall (Figure 1B and 1C). During panendoscopy the gigantic pedunculated tumor with origin in the left piriform sinus could not be sufficiently removed. Consequently, tumor resection was then performed via temporary esophagotomy (Figure 2). Macroscopically, the tumor measured $17.5 \times 7 \times 4.2 \mathrm{~cm}$ with a gray shiny surface, next to two smaller portions of $7 \times 4$ and $3.5 \times 2 \mathrm{~cm}$ with roughened surface. Cut surfaces were reddish-brownish, glassy and edematous, with adjacent lobulated yellowish parts. Histological examination of the tumor showed varying cell density, cell-poor sections with pronounced edematous myxoid stroma mixed with mature adipocytes with marginal small cell nuclei, subdivided by fibrous strands with hyperchromatic nuclei and adjacent sections with almost exclusively mature univacuolar fatty tissue. It was covered with dysplasia-free squamous epithelium (Figure 3A). There was no increased mitotic rate or necrosis. Immunohistochemical nuclear positivity for MDM2 and CDK4 was found (Figure 3B and 3C), FISH analysis showed strong amplification of the MDM2 gene (12q15, ratio of MDM2 to centrosome $=7.0$; Figure 3D). Thus, an atypical lipomatous tumor/welldifferentiated liposarcoma; FNCLCC grading: $1+0+0=1$ was diagnosed. After 10 days of feeding via gastric tube, oral nutrition was started again, and the patient was released from hospital 14 days postoperatively. No adjuvant therapy was necessary. In clinical follow-up and MRI control 6 months after surgery no hints for recurrence could be revealed, the patient is symptom-free.

\section{Case 2}

A 77-year old male patient presented in March 2017 with slight dysphagia and globus sensation. No dyspnea was reported.

${ }^{\star}$ Correspondence to: Maria Köping, Department of Oto-Rhino-Laryngology, Plastic, Aesthetic and Reconstructive Head and Neck Surgery, University Hospital Würzburg, Josef-Schneider-Strasse 11, D-97080 Würzburg, Germany, Tel: 0049931/201-21701; E-mail: koeping_m@ukw.de

Key words: liposarcoma, well-differentiated, hypopharynx

Received: May 30, 2019; Accepted: June 17, 2019; Published: June 20, 2019 


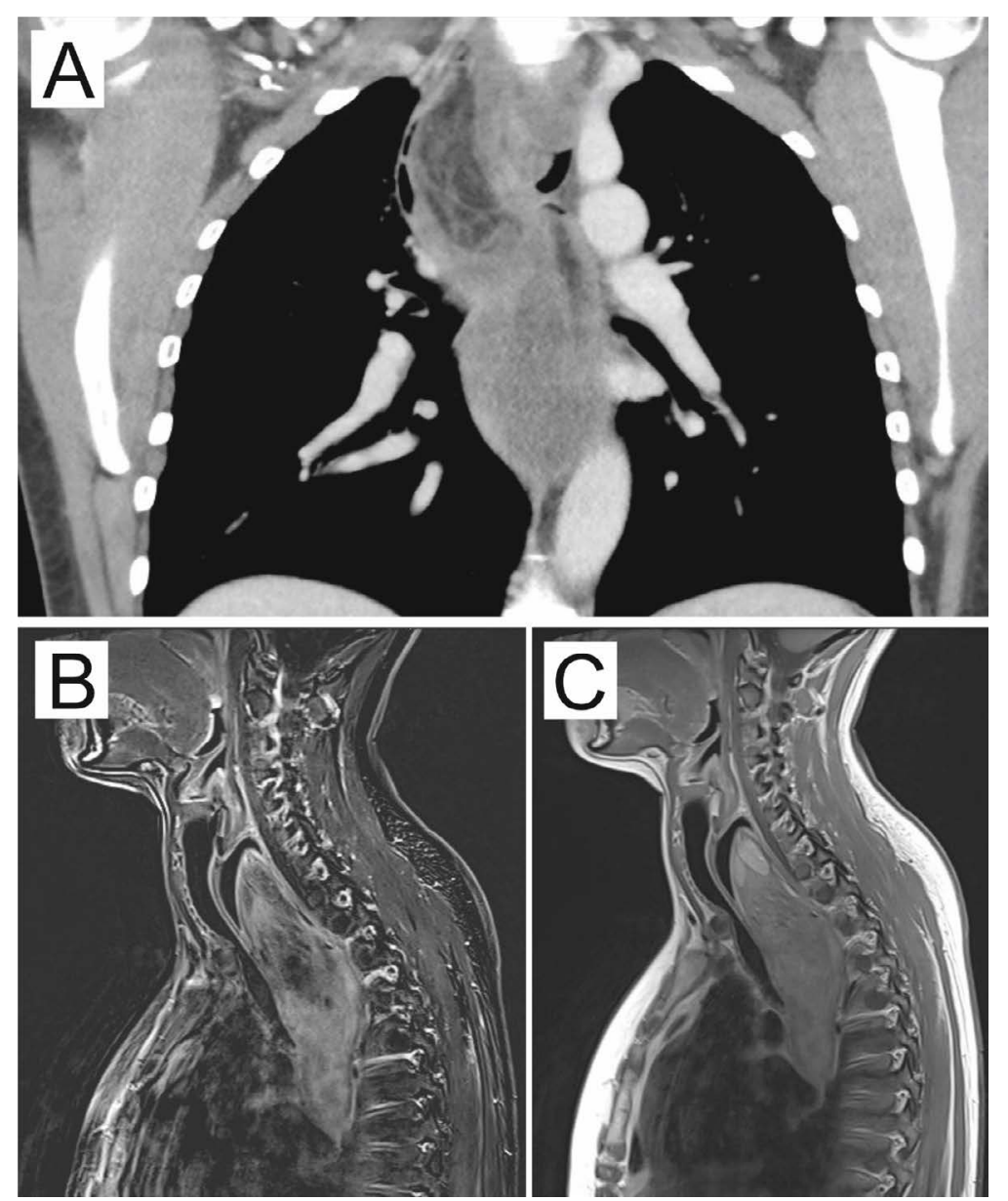

Figure 1. A) Postcontrast coronary CT scan, 5mm, showed a well-circumscribed low attenuation mass in the esophagus. T1-weighted sagittal subtraction MRI scan, B) and T1-weighted sagittal TSE scan, C) revealed an inhomogeneous tumor with fatty foci.
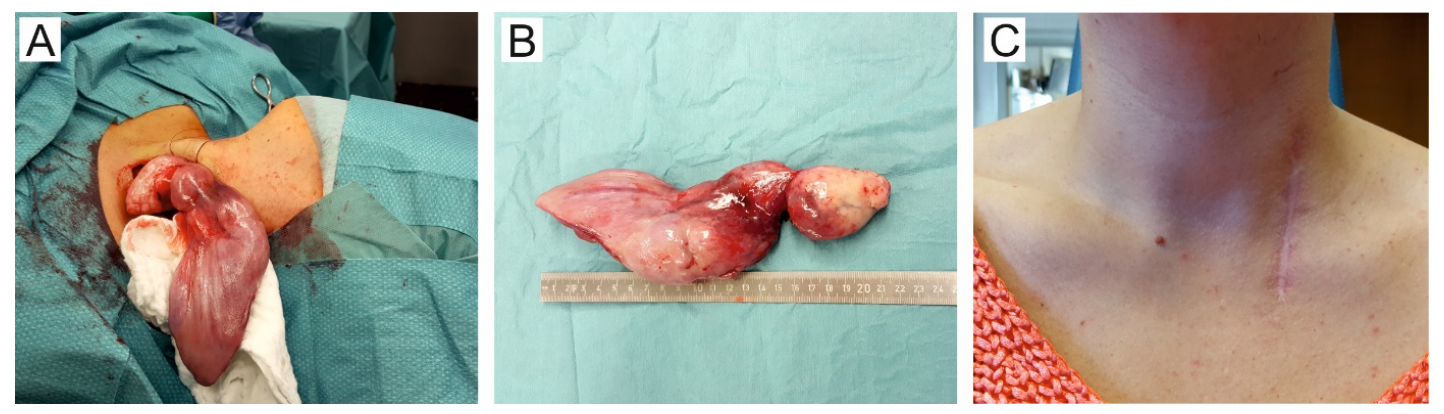

Figure 2. A) Intraoperative view during extraction over esophagotomy, B) The tumor after extraction measures $22 \mathrm{~cm}$, C) Control 6 months after surgery.

Transnasal endoscopy revealed a lobed tumor most likely originating from the hypopharynx which seemed soft and flexible and could be moved into a different position after swallowing (Figure 4). Vocal fold mobility was normal. Contrast enhanced CT scan revealed a polypoid, well-circumscribed mass in the hypopharynx and the dilated esophagus with mostly low tumor density and heterogeneous spots (Figure 5). In the following panendoscopy the pediculated tumor originating from the left hypopharyngeal wall was successively and completely removed. It had an extension of approximately $9 \times 5 \mathrm{~cm}$. Postoperative bleeding made a revision necessary and an extensive laryngeal edema required tracheotomy. Histologically, the polypoid light-colored reddish formation was covered with dysplasia-free squamous epithelium, consisting of fatty tissue and fibrous strands with hyperchromatic nuclei, mixed with scattered lymphocytes, plasma cells and mast cells. Immunohistochemical and cytogenetic testing revealed positivity for p16, MDM2 and CDK4, FISH amplification showed MDM2 gene amplification (12q15, ratio of MDM2 to centrosome = 5.0). A well-differentiated liposarcoma/atypical lipomatous tumor was diagnosed. Tracheostomy closure was performed after regular MRI and clinical follow-up. MRI examination after 6 months also revealed no suspicion of a tumor recurrence, the patient was symptom-free.

\section{Discussion}

Liposarcomas are the most frequent type of soft tissue sarcomas in adults [9]. The classification of Enzinger and Weiss is generally accepted which divides the entity of liposarcomas into 5 histopathological 

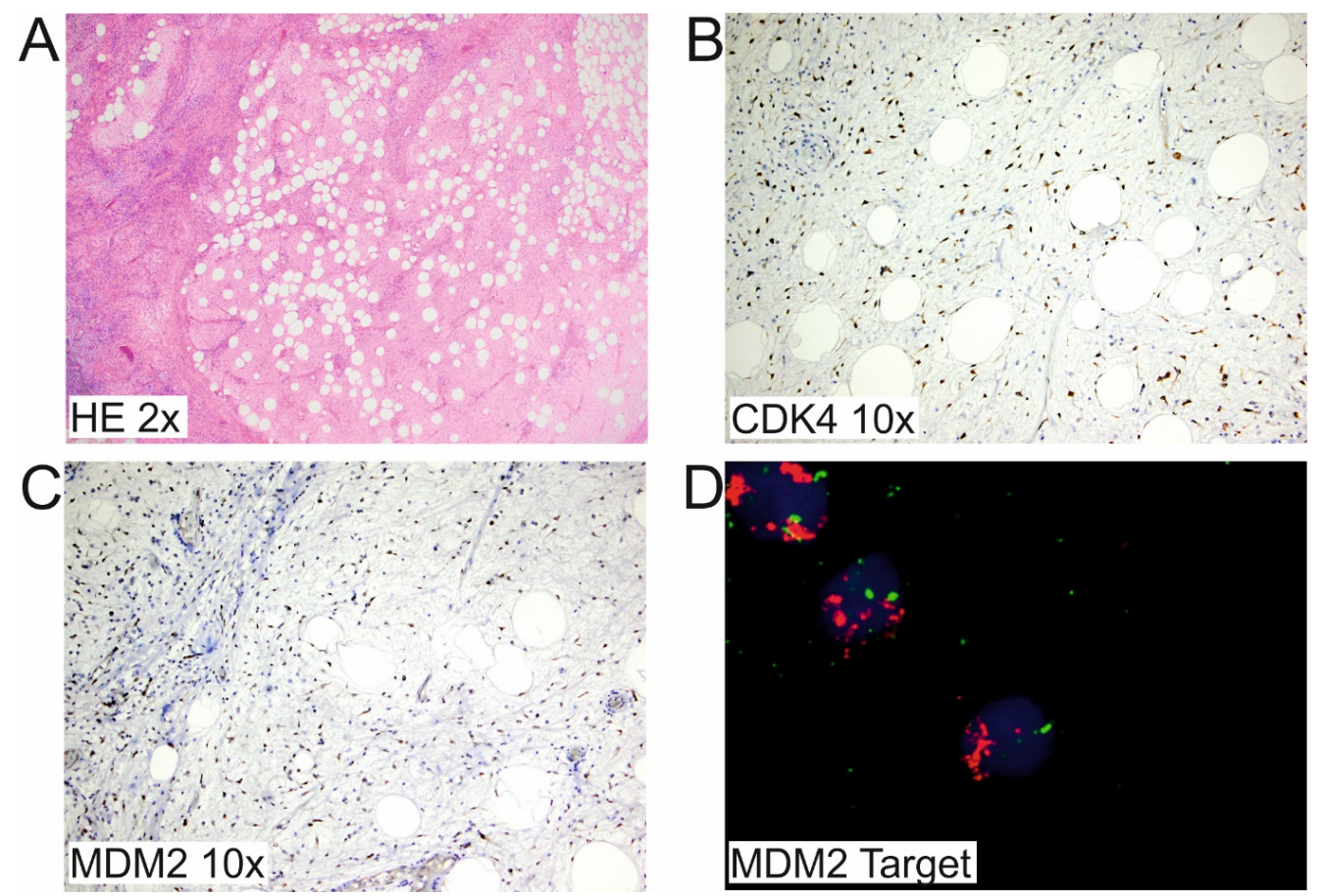

Figure 3. A) Foci of univacuolar variably sized adipocytes with mildly hyperchromatic nuclei separated by fibromyxoid areas (HE $\times 2$ ). Immunohistological examination showed nuclear positivity for CDK4 $(\mathrm{B}, \times 10)$ and MDM2 $(\mathrm{C}, \times 10)$. D) FISH assay with MDM2 target showed increased signals (red), corresponding to MDM2 gene amplification. (CDK4 cyclin-dependent kinase 4, MDM2 murine double minute-2)
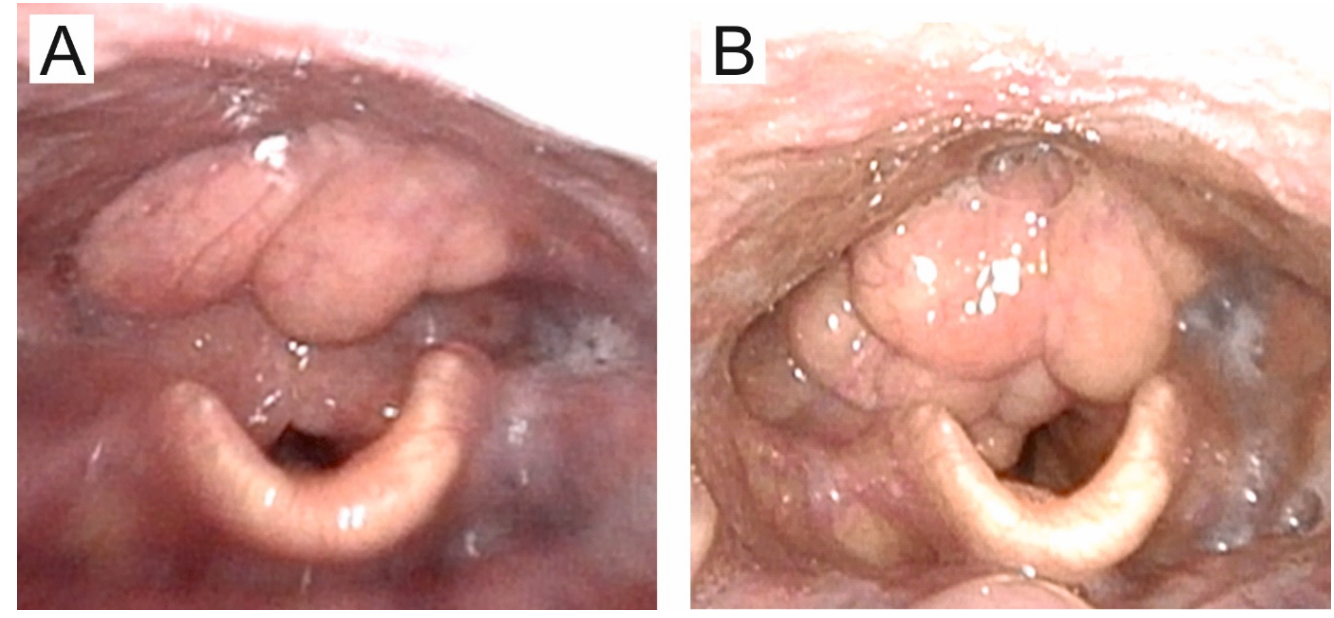

Figure 4. Preoperative transnasal endoscopic examination: the polypoid formation in the hypopharynx could be moved into a different position after swallowing (A/B).

subtypes: well-differentiated liposarcomas (synonymously called atypical lipomatous tumor) as most common ones, beside myxoid, pleomorphic, round cell and dedifferentiated liposarcoma [7].

The histological differentiation from other benign tumors like lipoma, fibrovascular polyps or fibroma can be challenging. MDM2 amplification by FISH is a helpful tool to confirm the diagnosis of a welldifferentiated liposarcoma $[3,8,10]$ as well as immunohistochemical staining with MDM2 and CDK 4.

Even though the well-differentiated liposarcoma is the most common form of liposarcoma, it is exceedingly rare in the hypopharynx with only few case reports in the literature [4]. In the majority of the cases, the tumor arises in the retroperitoneum and the extremities [3]. Liposarcomas demonstrate a peak occurrence in the sixth and seventh decade with a clear dominance of the male sex [4].
In the head and neck region, liposarcomas are typically of lowgrade malignancy and early stage, and have a better prognosis and overall survival compared to those found in other sites [11].

Due to localization and size of the tumor, the typical symptoms include dysphagia with or without weight loss, foreign body sensation or dyspnea. Sometimes, asphyxia can occur because of a sudden prolapse of the polypoid or pedunculated tumor formation into the airway [12]. Liposarcomas can reach gigantic dimensions before they cause any symptoms. The specimen in case 1 measured $18 \mathrm{~cm}$ but sizes up to $27 \mathrm{~cm}$ have been described [13].

Contrast-enhanced computed tomography (CT) of neck and chest shows a partly inhomogeneous low-density tumor with regular contours. In magnetic resonance imaging (MRI) liposarcomas present as a solid tumor with inhomogeneous signal intensity and foci of 

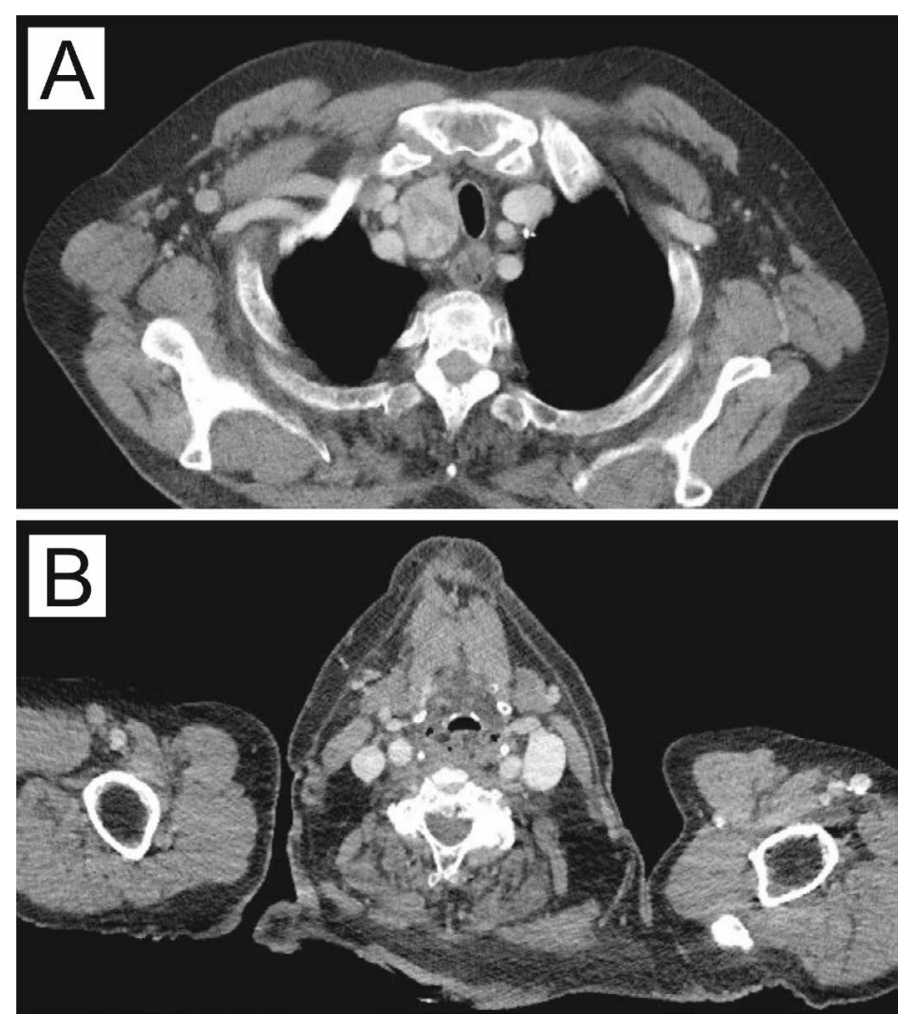

Figure 5. Contrast enhanced axial CT scan revealed a polypoid, well-circumscribed mass in the hypopharynx and the dilated esophagus. Tumor density was mostly low with heterogeneous spots $(\mathrm{A} / \mathrm{B})$

high signal intensity in T1-weighted images indicating a fatty mass. A correlation between intensity of fat signal and differentiation of liposarcoma is known [14].

Complete surgical removal is the treatment of choice in welldifferentiated liposarcomas. The two possible approaches are the endoscopic surgery or the transcervical approach (lateral pharyngotomy/esophagotomy). The endoscopic approach should be chosen if tumor size allows for an extirpation through the mouth. This method is less invasive with shorter hospitalization and faster subsequent oral nutrition. In gigantic liposarcomas the lateral pharyngotomy or esophagotomy is the gold standard. Procedures such as total laryngectomy should be limited to exceptional cases [3]. Nevertheless, wide and complete surgical excision is indispensable to ensure best outcome due to high risk of local recurrence up to $80 \%$. There is no evidence of the advantage of a postoperative radiotherapy or other adjuvant therapy so far [15,16]. Golledge et al. [5] also concluded that radiation does not add benefit to the management of welldifferentiated liposarcomas which have a very good prognosis. It may be useful in patients with higher grade liposarcomas or when surgical resection of a laryngeal liposarcoma is not practicable [17]. In the head and neck region well-differentiated (44.5\%) and myxoid type (35.5\%) of liposarcomas are the most prevalent subtypes and have the best outcome [11]. Prognosis in these low-grade subtypes of liposarcomas is very good with an overall 5-year survival up to $100 \%$ [10]. In contrast to the higher-grade round cell, pleomorphic or dedifferentiated liposarcomas, these subtypes extremely rarely metastasize [16].

To that point, no distant metastasis is known for hypopharyngeal well-differentiated liposarcomas [10] and no nodal metastases have been described [16].

\section{Conclusion}

These case reports and literature review confirm that the welldifferentiated liposarcoma of the hypopharynx is a very uncommon tumor which can lead to serious life-threatening complications. Complete and wide resection is essential to avoid local recurrence, but long-term follow-up must be carried out. Nodal or distant metastases are extremely rare, and a postoperative radiation is not recommended in these low-grade tumors. Otorhinolaryngologists should be aware of THIS unusual entity in unclear proliferation in the hypopharyngeal area.

\section{References}

1. Enzinger FM, Weiss SW (1988) Soft tissue tumors. ( $2^{\text {nd }}$ edn), Mosby Yearbook, St. Louis: $719-860$

2. Dubin MR, Chang EW (2006) Liposarcoma of the tongue: case report and review of the literature. Head Face Med 2: 21. [Crossref]

3. Han Y, Yang LH, Liu TT, Wang J, Li H, et al. (2015) Liposarcoma of the larynx Report of a case and review of literature. Int J Clin Exp Pathol 8: 1068. [Crossref]

4. Cornivo A, Riva G, Sensini M (2016) Liposarcomas of the hypopharynx. A systematic review of the literature. J Health Soc Sci 1: 57-66.

5. Golledge J, Fisher C, Rhys-Evans PH (1995) Head and neck liposarcoma. Cancer 76 1051-1058. [Crossref]

6. Eyermann C, Raguin T, Hemar P, Debry C (2018) Well-differentiated, pedunculated liposarcoma of the hypopharynx. Eur Ann Otorhinolaryngol Head Neck Dis 135: 6365. [Crossref]

7. Enzinger FM, Weiss SW (1995) Soft tissue tumors. Mosby Yearbook. Inc., St. Louis 325-335

8. Kimura H, Dobashi Y, Nojima T, Nakamura H, Yamamoto N, et al. (2013) Utility of fluorescence in situ hybridization to detect MDM2 amplification in liposarcomas and their morphological mimics. Int J Clin Exp Pathol 6: 1306-1316. [Crossref]

9. Adelson RT, DeFatta RJ, Verret DJ, Shen Y (2006) Liposarcoma of the tongue. Case report and review of the literature. Head Face Med 85: 749. [Crossref]

10. Zhu H, Sun J, Wei S, Wang D, Brandwein M (2017) Well-Differentiated Laryngeal Hypopharyngeal Liposarcoma in the MDM2 Era. Report of Three Cases and Literature Review. Head Neck Pathol 11: 146-151. [Crossref]

11. Gerry D, Fox NF, Spruill LS, Lentsch EJ (2014) Liposarcoma of the head and neck. Analysis of 318 cases with comparison to non-head and neck sites. Head Neck 36 : 393-400. [Crossref]

12. Caceres M, Steeb G, Wilks SM, Garrett Jr HE (2006) Large pedunculated polyps originating in the esophagus and hypopharynx. Ann Thorac Surg 81: 393-396. [Crossref]

13. Takiguchi G, Nakamura T, Otowa Y, Tomono A, Kanaji S, et al. (2016) Successful resection of giant esophageal liposarcoma by endoscopic submucosal dissection combined with surgical retrieval. A case report and literature review. Surg Case Rep 2: 1081. [Crossref]

14. Chung JJ, Kim MJ, Kim JH, Lee JT, Yoo HS et al. (2003) Imaging findings of giant liposarcoma of the esophagus. Yonsei Med J 44: 715-718. [Crossref]

15. Gritli S, Khamassi K, Lachkhem A, Touati S, Chorfa A, et al. (2010) Head and neck liposarcomas. A 32 years' experience. Auris Nasus Larynx 37: 347-351. [Crossref]

16. McCulloch TM, Makielski KH, McNutt MA (1992) Head and Neck Liposarcoma. A Histopathologic Reevaluation of Reported Cases. Arch Otolaryngol Head Neck Surg 118: 1045-1049. [Crossref]

17. Powitzky R, Powitzky ES, Garcia R (2007) Liposarcoma of the larynx. Ann Otol Rhinol Laryngol 116: 418-424. [Crossref]

Copyright: C2019 Köping M. This is an open-access article distributed under the terms of the Creative Commons Attribution License, which permits unrestricted use, distribution, and reproduction in any medium, provided the original author and source are credited. 